\title{
Polynomial Approximations to Finitely Oscillating Functions
}

\author{
By William J. Kammerer
}

1. Introduction. Chandler Davis [1] established the following theorem: If $v_{0}, v_{1}, \cdots, v_{n}$ are real numbers such that $v_{0}>v_{1}, v_{1}<v_{2}, v_{2}>v_{3}, \cdots$, then there exists a unique polynomial $P$ of degree $n$ and a set of points $y_{0}, y_{1}, \cdots, y_{n}$ such that

$$
\begin{array}{cr}
P\left(y_{i}\right)=v_{i} & i=0,1, \cdots, n \\
P^{\prime}\left(y_{i}\right)=0 & i=1,2, \cdots, n-1 \\
0=y_{0}<y_{1}<\cdots<y_{n}=1 . &
\end{array}
$$

The main result of this paper is an algorithm for the calculation of this polynomial, which is first motivated by an independent proof for the existence of $P$.

A function $f$ is said to be finitely oscillating if it has at most a finite number of relative extrema. The following mode of approximating a continuous finitely oscillating function $f$ in the uniform norm so that the oscillations are preserved, is discussed: first obtain a polynomial $P$ of minimal degree which has the same variation as $f$ and then obtain an increasing polynomial $Q$ such that $P(Q)$ agrees with $f$ at all its relative extrema. Two theorems are given in the last section, to show that this method of approximation is always possible.

2. Proof of Theorem. Let $D$ and $D_{0}$ denote the set of all $n+1$ tuples $X=\left(x_{0}, x_{1}, \cdots, x_{n}\right)$ such that $0=x_{0}<x_{1}<\cdots<x_{n}=1$ and $0=x_{0}<x_{1}<$ $\cdots<x_{n} \leqq 1$ respectively. Let $P$ denote the class of all polynomials $p$ of degree $n$, for which there exists an element $X \in D$, such that $p\left(x_{i}\right)=v_{i}(i=1,2, \cdots, n)$. A polynomial $p \in \&$ can be written in the following form:

$$
\begin{aligned}
p(x)=\left[x_{0}\right]+\left[x_{0}, x_{1}\right](x- & \left.x_{0}\right) \\
& +\cdots+\left[x_{0}, x_{1}, \cdots, x_{n}\right]\left(x-x_{0}\right) \cdots\left(x-x_{n-1}\right)
\end{aligned}
$$

where the bracket function is defined by

$$
\begin{gathered}
{\left[x_{i}\right]=v_{i}} \\
{\left[x_{i}, x_{i+1}, \cdots, x_{i+k}\right]=\frac{\left[x_{i+1}, \cdots, x_{i+k}\right]-\left[x_{i}, \cdots, x_{i+k-1}\right]}{x_{i+k}-x_{i}} .}
\end{gathered}
$$

LEMMA 2.1 If $X \in D_{0}$ and $n \geqq 1$ then $[X]>0$ for $n$ even and $[X]<0$ for $n$ odd.

Proof. The proof proceeds by induction. The lemma can be shown to hold for $n=1$ by direct computation. Assuming it true for $n=k-1$, one has $\left[x_{0}, x_{1}, \cdots\right.$, $\left.x_{k-1}\right]<0(>0)$ and $\left[x_{1}, x_{2}, \cdots, x_{k}\right]>0(<0)$ for $k$ even (odd). The lemma is therefore true for $n=k$ by (4).

Received March 11, 1960. This work was supported in part by the Office of Ordnance Research, U. S. Army. 
LEMMA 2.2. a) If $n$ is even (odd), the function $[X]$ assumes its minimum (maximum) in $D$.

b) Let $|[X]|$ attain its minimum value for $X \varepsilon D$, at $Y=\left(y_{0}, y_{1}\right.$, $\left.\cdots, y_{n}\right)$. Then the polynomial $p\left(y_{k}\right)=v_{k}(k=0,1, \cdots, n)$ is the desired polynomial $P$.

Proof. By lemma 2.1, $[X]$ is of constant sign in $D$. The function $|[X]|$ approaches infinity as $X$ approaches the boundary of $D$, and is continuous in the compact set $x_{0}+\epsilon \leqq x_{1}, x_{1}+\epsilon \leqq x_{2}, \cdots, x_{n-1}+\epsilon \leqq x_{n}$ for arbitrarily small $\epsilon>0$.

The following notation is introduced, to simplify the proof of part $b$ :

$$
\begin{aligned}
& \alpha(p, i)=\min \{x: x \varepsilon I(p, i)\} \\
& \beta(p, i)=\max \{x: x \in I(p, i)\}
\end{aligned}
$$$$
i=0,1, \cdots, n
$$

where

$$
\begin{gathered}
I(p, i)=\left\{x:\left[\begin{array}{l}
p(x) \geqq v_{i} \text { if } v_{i}>v_{i+1} \\
p(x) \leqq v_{i} \text { if } v_{i}<v_{i+1}
\end{array}\right] \text { and } x_{i-1}<x<x_{i+1}\right\} \\
i=1,2, \cdots, n-1 \quad I(p, 0)=0 \quad I(p, n)=1 \quad \text { and } p \varepsilon P .
\end{gathered}
$$

Let $Y$ be a vector in $D$ at which $|[X]|$ attains its minimum and let $p$ be the polynomial in o which satisfies $p\left(y_{i}\right)=v_{i}$. If $p$ does not satisfy (2), then there exists an integer $k$, such that $\alpha(p, k) \neq \beta(p, k)$. Let $x_{k}=\frac{1}{2}\{\alpha(p, k)+\beta(p, k)\}$ and consider the following two polynomials

$$
\begin{aligned}
& p(x)=h(x)+[Y] g_{k}(x, Y) \\
& p_{1}(x)=h(x)+\left[y_{0}, \cdots y_{k-1}, x_{k}, y_{k+1}, \cdots, y_{k}\right] g_{k}(x, Y)
\end{aligned}
$$

where $h(x)$ is a polynomial of degree $n-1$, and

$$
g_{k}(x, Y)\left(x-y_{k}\right)=\prod_{i=0}^{n}\left(x-y_{i}\right)
$$

By construction one has $\left|p_{1}\left(x_{k}\right)\right|<\left|p\left(x_{k}\right)\right|$. Investigation of the possible cases contradicts the hypothesis that $Y$ is a relative extrema of $[X]$.

It should be observed that the above theorem and proof are valid for polynomials of the form $\sum_{k=0}^{n} a_{k} f(x)^{k}$ where $f$ is a strictly increasing differentiable function on $[0,1]$. The proof is identical except for notation.

\section{An Iterative Procedure.}

Step 1. Choose an arbitrary element $X_{1}=\left(x_{0}{ }^{1}, x_{1}{ }^{1}, \cdots, x_{n}{ }^{1}\right)$ in $D$.

Step 2. With one of the standard interpolating formulas construct the polynomial $p_{1} \varepsilon P$, such that $p_{1}\left(x_{k}{ }^{1}\right)=v_{k}, k=0,1, \cdots ; n$.

Step 3. Determine the vector $X_{2}=\left(x_{0}{ }^{2}, x_{1}{ }^{2}, \cdots, x_{n}{ }^{2}\right)$ in $D$ such that $p_{1}{ }^{\prime}\left(x_{1}{ }^{2}\right)=0$ for $i=1,2, \cdots, n-1$.

We now have a new element $X_{2} \varepsilon D$. To obtain $p_{2}$, repeat this process beginning with step 2, using $X_{2}$ in place of $X_{1}$ and making the obvious change in subscripts. 
Continuing this procedure, we obtain a recursive process for obtaining a sequence $\left\{p_{i}\right\}$.

THEOREM 3.1. The sequence $\left\{p_{i}\right\}$ converges uniformly to $P$.

Proof. The proof will proceed by a series of lemmas.

LEMMA 3.2. If $p \in \mathcal{P}$, then

$$
\begin{array}{r}
{\left[x_{0}, \cdots, x_{k-1}, \alpha(p, k), x_{k+1}, \cdots, x_{n}\right]=\left[x_{0}, \cdots, x_{k-1}, \beta(p, k), x_{k+1}, \cdots, x_{n}\right]} \\
k=1,2, \cdots, n-1 .
\end{array}
$$

Proof. By (3), $p$ can be written in the following two forms

$$
\begin{aligned}
& p(x)=h(x)+\left[x_{0}, \cdots, x_{k-1}, \alpha(p, k), x_{k+1}, \cdots, x_{n}\right] g_{k}(x, X) \\
& p(x)=h(x)+\left[x_{0}, \cdots, x_{k-1}, \beta(p, k), x_{k+1}, \cdots, x_{n}\right] g_{k}(x, X)
\end{aligned}
$$

where $h(x)$ is a polynomial of degree $n-1$ and $g_{k}(x, X)$ is defined as in $(5)$. To obtain the desired result, subtract (6) from (7).

LEMMA 3.3. Let $p$ be an element of $\beta$ and let $N(p)=\max _{i}\left|m_{i}-v_{i}\right|$ where $m_{i}=\max |p(x)|$ for $x \varepsilon[\alpha(p, i), \beta(p, i)]$ and $i=1,2, \cdots, n-1$. Then the following inequalities hold for the sequence $\left\{p_{i}\right\}$ :

a) If $N\left(p_{i}\right) \neq 0$ then $\left|\left[X_{i+1}\right]\right|<\left|\left[X_{i}\right]\right|$

b) $\left|p_{i}(x)-p_{i+1}(x)\right| \leqq\left|\left[X_{i}\right]-\left[X_{i+1}\right]\right|$ for every $x \varepsilon[0,1 \mid$

c) $N\left(p_{i}\right) \leqq \max _{0 \leqq x \leqq 1}\left|p_{i}(x)-p_{i+1}(x)\right|$.

Proof. Let $i$ be any positive integer and let $\left\{h_{k}\right\}, k=1,2, \cdots, n-1$ be the polynomials of degree $n$ such that

$$
\begin{aligned}
h_{k}\left(x_{m}{ }^{i+1}\right) & =v_{m} & \text { for } m=0,1, \cdots, h \\
h_{k}\left(\beta_{m}\right) & =v_{m} & \text { for } m=k+1, \cdots, n
\end{aligned}
$$

where $\beta_{m}=\beta\left(p_{i}, m\right)$. Let $Z_{k}$ denote the vector $\left(x_{0}{ }^{i+1}, \cdots, x_{k}{ }^{i+1}, \beta_{k+1}, \cdots, \beta_{n}\right)$. Then $I\left(h_{k}, m\right) \subset I\left(h_{k+1}, m\right)$ for $m=k+2, \cdots, n$, for otherwise $h_{k}(x)-h_{k+1}(x)$ would be identically equal to zero, instead of having $n-1$ simple roots in $[0,1]$. As in the proof of lemma 2.2 , we have $\left|\left[Z_{0}\right]\right| \geqq\left|\left[Z_{1}\right]\right| \geqq \cdots \geqq\left|\left[Z_{n}\right]\right|$ with at least one of the inequalities being strict, since by assumption, $N\left(p_{i}\right) \neq 0$. This proves part $a$.

For $k=0,1, \cdots, n-1$ one has $\left|h_{k}(x)-h_{k+1}(x)\right|=\left|\left[Z_{k}\right]-\left[Z_{k+1}\right]\right|$. $\left|g_{k}\left(x, Z_{k}\right)\right| \leqq\left|\left[Z_{k}\right]-\left[Z_{k+1}\right]\right|$. Now apply the triangle law to obtain $b$.

The proof of part $c$ follows from the existence of an integer $k$, such that $N\left(p_{i}\right)=$ $\left|p_{i}\left(x_{k}^{i+1}\right)-p_{i+1}\left(x_{k}^{i+1}\right)\right|$.

To complete the proof of theorem 3.1, observe that lemmas 2.1 and 3.3.a imply that $\left\{\left[X_{i}\right]\right\}$ is a Cauchy sequence. Lemma 3.3.b implies $\left\{p_{i}\right\}$ also forms a Cauchy sequence in the uniform norm on $[0,1]$ and therefore convergent to a polynomial $P$ of degree $n$. Part $c$ of lemma 3.3 implies that $P$ satisfies conditions 1 and 2 .

This iteration can be carried out by the use of standard subroutines available in most computer libraries, and from all empirical evidence the convergence seems quite rapid. To illustrate, we shall calculate the third-degree Chebyshev polynomial on $[0,1]$ by the use of this method, starting with $X_{1}=(0, .25, .5,1)$. 


$$
\begin{gathered}
p_{1}(x)=1-22 x+68 x^{2}-48 x^{3} \\
X_{2}=(0, .20723911, .73720533,1) \\
p_{2}(x)=1-18.400541 x+48.970905 x^{2}-32.570364 x^{3} \\
X_{3}=(0, .24979357, .75256767,1) \\
p_{3}(x)=1-18.000432 x+48.002138 x^{2}-32.001707 x^{3} \\
X_{4}=(0, .24999339, . .74999783,1) \\
p_{4}(x)=1-17.999999 x+47.999999 x^{2}-31.999999 x^{3}
\end{gathered}
$$

\section{Approximation by Composition.}

Theorem 4.1. Let $X=\left(x_{0}, x_{1}, \cdots, x_{n}\right), Y=\left(y_{0}, y_{1}, \cdots, y_{n}\right)$ be any two elements in $D$. Then there exists a polynomial $Q$ such that $Q^{\prime}(x) \geqq 0$ on $[0,1]$ and $Q\left(x_{i}\right)=y_{i}, i=0,1, \cdots, n$.

Proof. Define the elements $Z_{1}$ and $Z_{2}$ in $D$ as follows:

$$
\begin{aligned}
& Z_{1}=\left(z_{0}{ }^{1}, z_{1}{ }^{1}, \cdots, z_{n}{ }^{1}\right)=\left(y_{0}, \frac{1}{4}\left(y_{2}-y_{1}\right)+y_{1}, \frac{1}{4}\left(y_{3}-y_{2}\right)+y_{2}, \cdots, y_{n}\right) \\
& Z_{2}=\left(z_{0}{ }^{2}, z_{1}{ }^{2}, \cdots, z_{n}{ }^{2}\right)=\left(y_{0}, \frac{1}{4}\left(y_{1}-y_{0}\right)-y_{1}, \frac{1}{4}\left(y_{2}-y_{1}\right)-y_{2}, \cdots, y_{n}\right) .
\end{aligned}
$$

Let $f_{i}$ for $i=1,2,3, \cdots, 2^{n-1}$ be distinct piecewise linear functions, which are linear on the intervals $\left[y_{i-1}, y_{i}\right] i=1,2, \cdots, n$ and such that $f_{i}\left(x_{k}\right)=z_{k}{ }^{1}$ or $z_{k}{ }^{2}, k=0$, $1, \cdots, n$. Define $\epsilon=\min _{i} \min _{k}\left|z_{i}^{k}-y_{i}\right|$ for $i=1,2, \cdots, n-1, k=1,2$. Using the fact that the Bernstein polynomials of a continuous increasing function are increasing and uniformly convergent, there exist increasing polynomials $Q_{i}(x)$ on $[0,1]$ such that $\left|f_{i}(x)-Q_{i}(x)\right|<\frac{1}{2} \epsilon$ for $i=1,2, \cdots, 2^{n-1}$ (see Lorentz [7] p. 20-23). The vector $Y$ is contained in the convex hull of the vectors $\left(Q_{i}\left(x_{0}\right)\right.$, $\left.Q_{i}\left(x_{1}\right), \cdots, Q_{i}\left(x_{n}\right)\right), i=1,2, \cdots, 2^{n-1}$, and therefore there exists a convex linear combination of the $Q_{i}$ 's which will give rise to a desired polynomial $Q$.

THEOREM 4.2. Let $f$ be a continuous finitely oscillating function on $[0,1]$ and let $\varepsilon>0$ be given. Then there exist polynomials $P(y)$ and $Q(x)$, such that

a) $f(x)$ and $P(y)$ are equal at their corresponding relative extrema. At the relative extrema of $f$,

$$
P(Q)=f \text { and } \frac{d P(Q)}{d x}=0 .
$$

b) The polynomial $Q$ is increasing and $|f(x)-P(Q(x))|<\epsilon$ on $[0,1]$.

Proof. Let the partition $X=\left(x_{0}, x_{1}, \cdots, x_{n}\right)$ be the points of the relative extrema of $f$ on $[0,1]$. By a previous theorem, there exists a polynomial $P(y)$ and a partition $Y=\left(y_{0}, y_{1}, \cdots, y_{n}\right)$ such that $P\left(y_{i}\right)=f\left(x_{i}\right), i=0,1, \cdots, n$ and $P^{\prime}\left(y_{i}\right)=0, i=1,2, \cdots, n-1$. Let $X^{\prime}$ be a refinement of the partition $X$ which satisfies the following condition $x_{i}=x_{i 1}<x_{i 2}<\cdots<x_{i N_{i}}=x_{i+1}$ such that $N_{i}\left|f\left(x_{i j}\right)-f\left(x_{i j+1}\right)\right|=\left|f\left(x_{i}\right)-f\left(x_{i+1}\right)\right| \leqq N_{i} \in$ for $i=0,1, \cdots, n-1$, $j=1,2, \cdots, N_{i}$. Define $Y^{\prime}$ to be the refinement of the partition $Y$ such that $y_{i j} \varepsilon\left[y_{i}, y_{i+1}\right]$ and $f\left(x_{i j}\right)=P\left(y_{i j}\right)$ for $i=0,1, \cdots, n-1, j=1,2, \cdots, N_{i}$. By theorem 4.1 there exists an increasing polynomial $Q(x)$ on $[0,1]$, such that $Q\left(x_{i j}\right)=$ $y_{i j}$, and therefore by construction $|p(Q(x))-f(x)|<\epsilon$ on $[0,1]$.

As a concluding remark, let $C$ represent the class of all composite polynomials of the, form $P(Q)$, where both $P$ and $Q$ are of degree greater than unity and $Q^{\prime} \geqq 0$ 
on $[0,1]$. Not every polynomial of degree four or larger can be so written (see H. Levi [6]). However, since every continuous function on $[0,1]$ can be uniformly approximated by a polynomial, (i.e., a finitely oscillating function) one finds that the completion of $C$ in the uniform norm on $[0,1]$ is the set of all contimous functions on $[0,1]$.

The question of the existence of variation preserving approximations arose from the investigation of syntonic functions by Professor P. C. Hammer in [2] and [3]. I would also like to thank the referee for pointing out that the ideas in references $[4],[5]$ and [8] bear a relation to the problem treated here.

Georgia Institute of Technology

Atlanta, Georgia

1. Chanderer Davis, "Extrema of a polynomial," Amer. Math. Monthly, v. 64, 1957, p. $679-680$.

2. P. C. HAMMER "Syntonicity of functions and the variation functional," Rend. Circ. Mat. Palermo, Serie II, Tomo VIII, Anno 1959, p. 145-151.

3. P. C. HAMmer \& J. C. Holladay, "Functions of restricted variation," Rend. Circ. Mat. Palermo, Serie II, Tomo VIII, Anno 1959, p. 102-114.

4. R. S. JoHNSON, "On monosplines of least deviation," Trans. Amer. Math. Sor., v. 96, 1960, p. $458-477$.

5. A. J. Kempner, "On the shape of polynomial curves," Tohoku Math. J., Part I, v. 37, 1933, p. 347-352; Part II, v. 42, 1936, p. 318-330.

6. H. LEvI, "Composite polynomials with coefficients in an arbitrary field of characteristic zero," Amer. J. Math., v. 64, 1942, p. 389-400.

7. G. G. LoRenth, Bernstein Polynomials, University of Toronto Press, 1953.

8. G. R. MACLANE, "Concerning the uniformization of certain Riemann surfares allied to the inverse-cosine and inverse-gamma surfaces," Trans. Amer. Math. Soc., v. 62, 197\%, p. 99-113. 